\title{
Brecha salarial entre hombres y mujeres en el mercado laboral de los Estados miembros del MERCOSUR, período 1991-2020
}

\section{Wage gap between men and women in the labor market of the MERCOSUR member states, 1991-2020 period}

\author{
Ruth Romina Arleth Romero Vian ${ }^{\circledR}$, Marcelo Mathías Sánchez Zárate ${ }^{\mathbb{1}}$, \\ Mathías Federico González Acosta ${ }^{10}$, Antonella Cabral
}

\begin{abstract}
Resumen
El tema de estudio de la investigación es la brecha salarial entre hombres y mujeres, concretamente en el mercado laboral de los Estados miembros del MERCOSUR y a su vez cómo la desigualdad de género tiene implicancias directas en la misma. Se ha examinado la brecha salarial ajustada por hora trabajada en cada uno de los países de estudio. La brecha salarial se ha manifestado a lo largo de los treinta años en que se enmarca esta investigación arrojando como resultado una menor brecha salarial en los países con una política de género más rígida. Las recomendaciones finales se engloban en fomentar políticas que incentiven la creación de proyectos con el fin de beneficiar la entrada de la mujer en el mercado laboral y que su permanencia en ella se dé con igualdad de condiciones.

Palabras clave: brecha, desigualdad, género, MERCOSUR.
\end{abstract}

\begin{abstract}
The subject of the research is the wage gap between men and women, specifically in the labor market of the MERCOSUR member states, and how gender inequality has direct implications on it. The adjusted wage gap per hour worked has been examined in each of the countries under study. The wage gap has manifested itself over the thirty years in which this research is framed, resulting in a smaller wage gap in the countries with a more rigid gender policy. The final recommendations are focused on promoting policies that encourage the creation of projects to benefit women's entry into the labor market and ensure that they remain in it under equal conditions.

Keywords: gap, inequality, gender, MERCOSUR.
\end{abstract}

\section{Introducción}

Toda sociedad está constituida tanto por hombres como mujeres y también es evidente que existen diferencias con respecto al relacionamiento entre ambos. Con el transcurso de los años estas diferencias han ido mermando hasta la actualidad, pero siguen siendo un gran reto a nivel mundial sobre todo en el ámbito
1 Universidad Nacional de Asunción, Facultad de Ciencias Económicas. San Lorenzo, Paraguay.

Correspondencia a: rromeroogo@alumnos.eco.una.py

Recibido:

28 de setiembre de 2021

Aceptado:

4 de noviembre de 2021

Doi:

https://doi.org/10.54549/ky.6e.2021.54

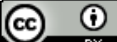

Artículo publicado en acceso abierto bajo la Licencia Creative Commons.

Cita:

Romero Vian, R. R. A.,

Sánchez Zárate, M. M.

González Acosta, M. F., \& Cabral, A. (2021). Brecha salarial entre hombres y mujeres en el mercado laboral de los Estados miembros del MERCOSUR, período 19912020. Kera Yvoty: reflexiones sobre la cuestión social, 6(número especial), 54-61. https://doi.org/10.54549/ ky.6e.2021.54 
socioeconómico dando un especial énfasis en el mercado laboral.

Después de la primera mitad del siglo $\mathrm{XX}$, las mujeres se introducen al mercado laboral, involucrándose en ella cada vez más, hasta entonces la discriminación de sexo las había excluido de dicho mercado, no quedando otra alternativa más que optar por los trabajos en los hogares no remunerados. Es por ello que su incursión al mundo laboral ha supuesto un importante avance, esencialmente en su economía autónoma (Artza et al., s. f.). Sin embargo, esta incursión se ha llevado a cabo con desventaja, incluso pudiéndose observar a día de hoy en mayor desempleo femenino, una mayor informalidad y una fuerte concentración en determinadas ocupaciones como también en la participación en la parte baja en las estructuras jerárquicas de las empresas, siendo la brecha salarial el manifiesto más evidente de la desigualdad de género.

La discriminación en el mercado laboral es una situación en la que personas que son igualmente productivas son tratadas de manera desigual, situación que está relacionada con sus características observables como la raza, etnia o sexo
(Altonji \& Blank, 1999).

También es fundamental mencionar a Mill quien en el año 1869 fue el primer economista en enfocarse exclusivamente en el género dando prioridad sobre la situación social de las mujeres. Es así que la motivación de la investigación es la explicación de las causas que se encuentran subyacentes a la desigualdad de género en el mercado laboral de los Estados miembros del MERCOSUR, lo que supone a su vez la exploración del conflicto del rol de género arraigado desde hace décadas en la historia humana, además del conocimiento mismo que implica un tema que abarca múltiples consecuencias y afecta a un entorno social, cultural y económico.

\section{Objetivo General}

Analizar la evolución de las brechas salariales por género de los Estados miembros del MERCOSUR, período 19912020.

\section{Metodología}

La investigación se encuentra dentro de la modalidad bibliográfica documental, recurriendo a la utilización de materiales bibliográficos tales como: libros, artículos

Figura 1. Evolución del Ranking de Paridad de Género, Período 2006-2020

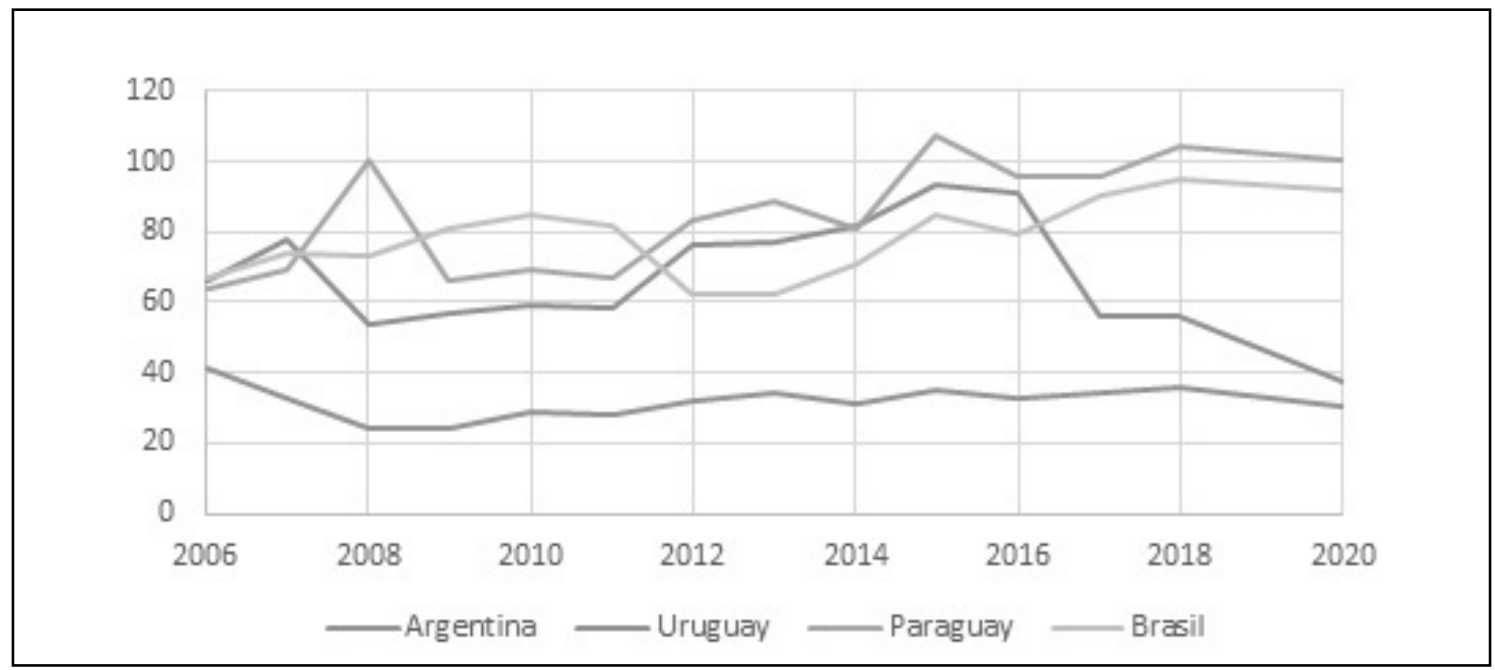

Fuente. Elaboración propia hecha a partir de los datos obtenidos de la Global Gender Gap Report. 
Romero Vian et al. Brecha salarial entre hombres y mujeres en el mercado laboral de los Estados miembros del MERCOSUR, período 1991-2020

Figura 2. : Evolución de la Brecha Salarial en Paraguay, Período 1995-2018

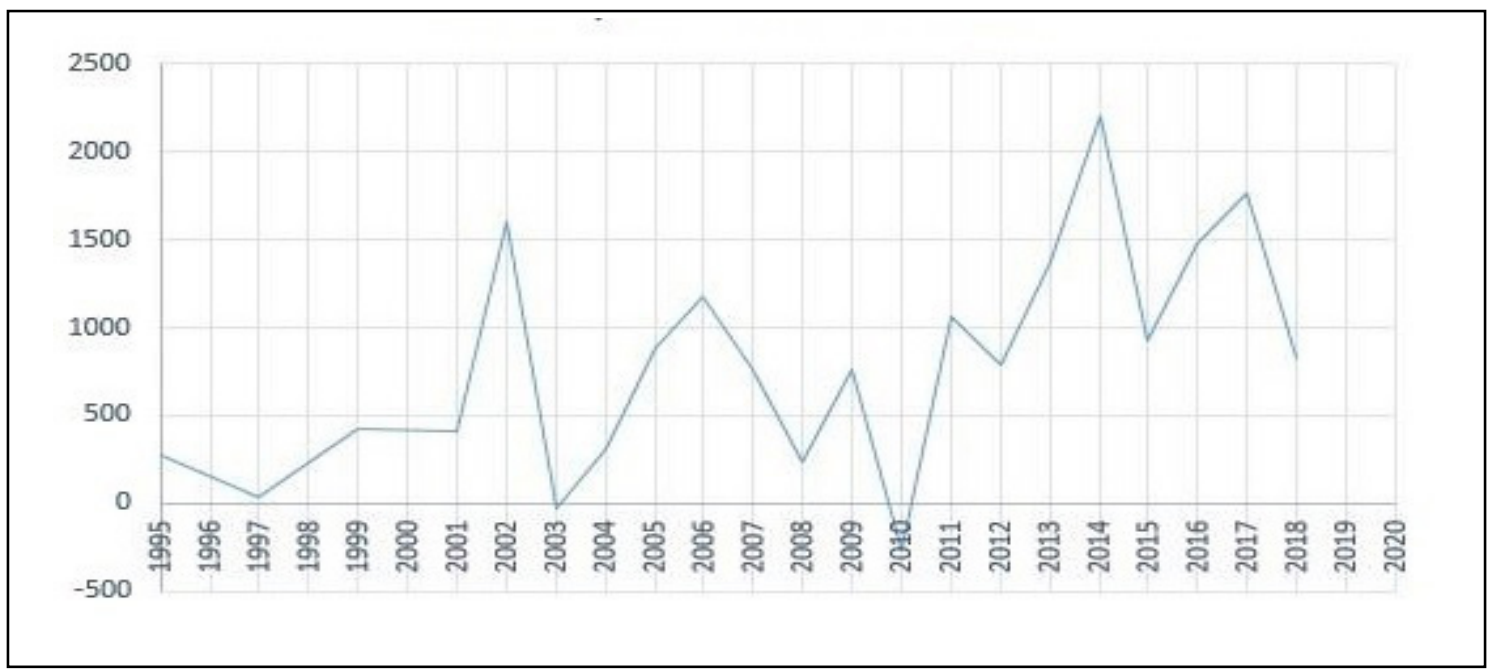

Fuente. Elaboración propia hecha a partir de la Encuesta Permanente de Hogares (EPH) de Paraguay. Base de Datos Socioeconómicos para América Latina y el Caribe (SEDLAC).

científicos, revistas científicas, sitios web oficiales, y materiales documentales como: datos oficiales de la Dirección General de Estadísticas, Encuestas y Censos (DGEEC) utilizando como base de datos la Encuesta Permanente de Hogares (EPH); Instituto Nacional de Estadística y Censos utilizando como base datos la Encuesta Permanente de Hogares (EPH) de la República Argentina; Instituto Nacional de Estadística, haciendo uso de la base de datos de la Encuesta Continua de Hogares (ECH); Instituto Brasileño de Geografía y Estadística (IBGE) utilizando como base de datos la Pesquisa Nacional por Amostra de Domicílios (PNAD), se acudió también a datos oficiales del Banco Mundial (BM), además se pueden mencionar como ejemplos a los siguientes sitios web: Organización de las Naciones Unidas Mujeres (ONU Mujeres), Foro Económico Mundial (FEM) y Fondo Monetario Internacional (FMI), como también se utilizaron los informes de la Comisión Económica Para América Latina y El Caribe (CEPAL) y de la Organización Nacional del Trabajo (OIT).

El nivel de investigación que se ha empleado es el nivel descriptivo, pues se detallan las características de lo estudiado y se describen los hechos como son observados en la realidad del mercado laboral de los Estados Miembros del MERCOSUR.

El método utilizado es de investigación histórica con datos estadísticos históricos que consisten en varias cifras y números, además, del método histórico de la investigación para poder sintetizar la evolución de la brecha salarial en el mercado laboral de los Estados miembros a lo largo de la historia. También se utilizó el método sintético, llegando a una conclusión de manera general.

\section{Resultados y Discusión}

\section{1. Índice de Paridad de Género de los Estados miembros del MERCOSUR, un panorama general}

El Global Gender Gap Report (Informe de la Brecha Global de Género) es publicado anualmente desde el año 2006 por el World Economic Forum (Foro Económico Mundial, Figura 1) y analiza la división de los recursos y las oportunidades entre hombres y mujeres en 153 países. Por 
un lado, Paraguay ocupa el puesto $100^{\circ}$ en el ranking de brecha de género y en el gráfico se observa la evolución del puesto que ocupa el país en el mencionado informe, destacando que existen grandes diferencias salariales dentro del mercado laboral analizado.

Asimismo, Brasil, otro Estado Miembro del MERCOSUR ocupa el puesto $92^{\circ}$ en la edición 2020, entre 149 países clasificados. Cabe destacar que Brasil posee una tendencia ascendente observable en los últimos años.

En cuanto a Argentina, el mismo ocupa el puesto $30^{\circ}$ en la edición 2020 , posee una muy buena posición en el ranking en lo que a paridad de género refiere, fluctuando entre los puestos $28^{\circ}$ $41^{\circ}$, es decir la diferencia entre hombres y mujeres no son grandes si las comparamos con las del resto de los países analizados.

Por último, Uruguay se sitúa en el puesto $37^{\circ}$ del ranking de la brecha de género en el año 2020, es decir, han disminuido las diferencias salariales entre hombres y mujeres respecto a años anteriores.

\subsection{Evolución de la brecha salarial en el mercado laboral de los Estados miembros del MERCOSUR}

\section{Paraguay}

La inserción laboral de las mujeres se ha debido gracias a un cambio estructural enfocado a varios sectores del mercado laboral del Paraguay en las últimas dos décadas, una transición desde el sector primario hacia las industrias como también hacia los servicios públicos.

A pesar de la participación de las mujeres en el mercado laboral de Paraguay en el período 1995-2018 posee una tendencia creciente (aunque en los últimos años es notorio una desaceleración), la misma se encuentra constantemente por debajo de los hombres, siendo la brecha en

Figura 3. Evolución de la Brecha Salarial en Brasil, Período 1990-2015.

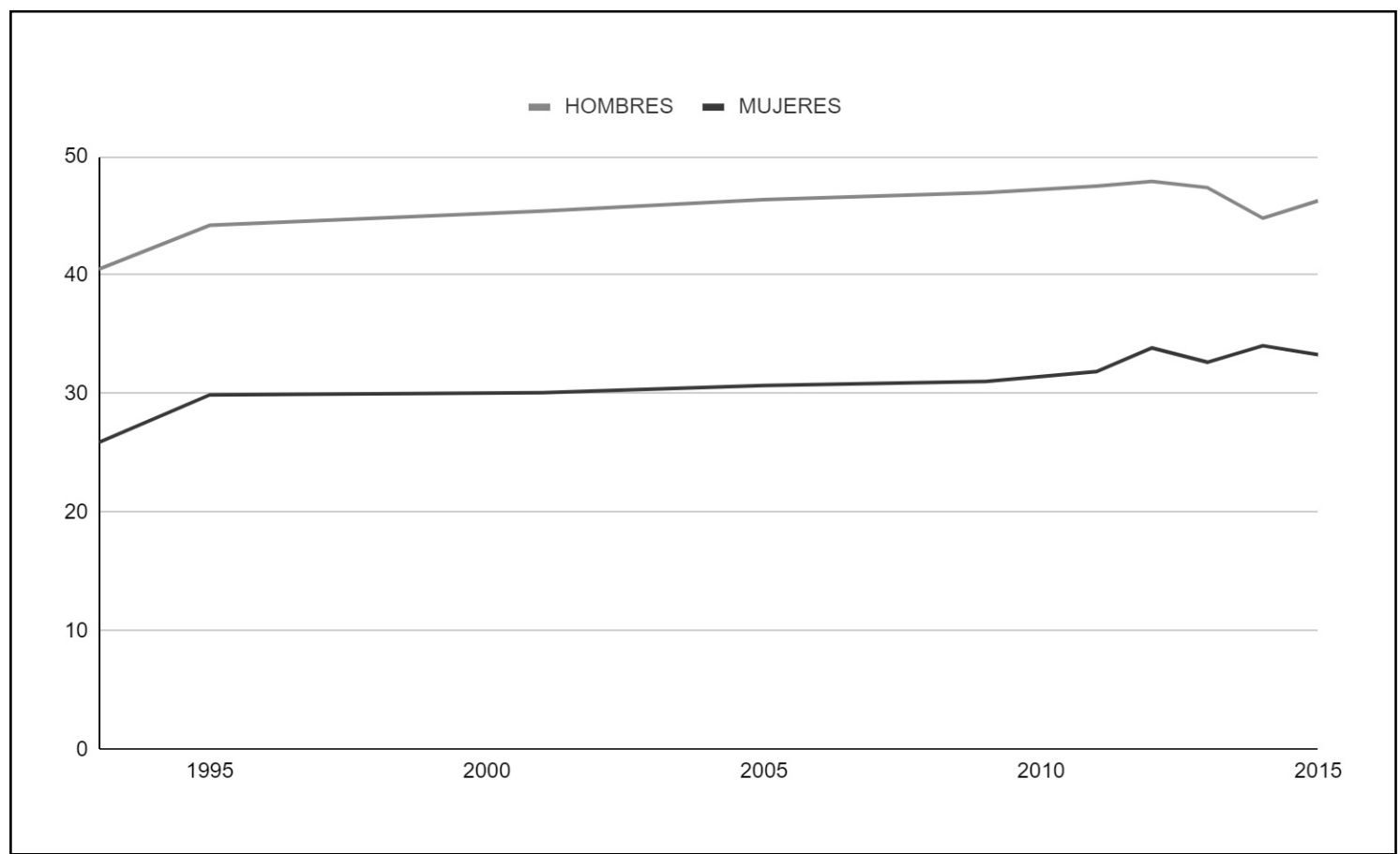

Fuente. Elaboración propia a partir de los datos obtenidos de la Pesquisa Nacional por Amostra de Domicílios (PNAD). 
Romero Vian et al. Brecha salarial entre hombres y mujeres en el mercado laboral de los Estados miembros del MERCOSUR, período 1991-2020

Figura 4. Evolución de la Brecha Salarial en Argentina, Período 1995-2019.

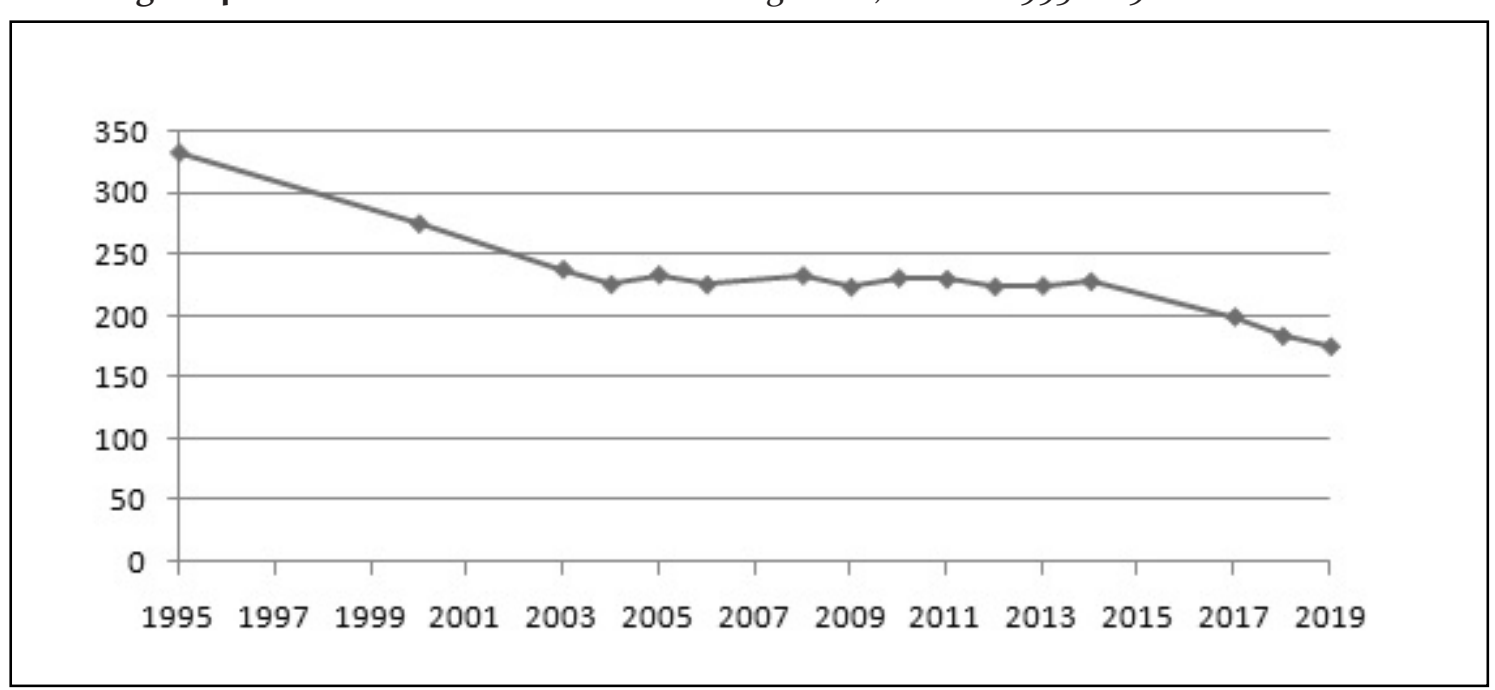

Fuente. Elaboración propia hecha a partir de los datos obtenidos de la Organización Internacional del Trabajo (OIT).

la participación laboral en el año 2015 de aproximadamente un $20 \%$ y todo ello ocurre bajo un contexto en el que los niveles de educación tanto de hombres como mujeres son similares (Serafini \& Egas, 2018).

Asimismo, existe una marcada brecha salarial entre hombres y mujeres, una brecha que es observable incluso en la actualidad, en el siguiente gráfico se analiza la evolución de la brecha salarial por género de la población de entre 25-64 años (Figura 2).

\section{Brasil}

Anteriormente los hombres actuaban como principal proveedor en el núcleo familiar, pero esto ha quedado como un concepto obsoleto. Con el transcurso del tiempo se ha observado que en el Brasil la participación de las mujeres en el mercado laboral ha ido aumentando considerablemente y con ello la cultura de la igualdad de género. Comúnmente las diferencias salariales en el país se les atribuyen a los altos costes que representan las mujeres, la cual es una significativa barrera para el acceso a mejores empleos.

Uno de los principales determinantes de la desigualdad salarial en Brasil está relacionado con las disparidades educacionales entre los que conforman la fuerza de trabajo (Freitas, 2015). De esta manera, parte de la brecha salarial por género debe ser explicada por los diferenciales productivos de la población, mientras que la otra parte se debe a factores discriminatorios. En las últimas décadas existieron cambios significativos en la composición del mercado de trabajo, principalmente en lo que se refiere a la disminución de la brecha salarial por género (Figura 3).

\section{Argentina}

Las reformas económicas y laborales iniciadas en 1991 en Argentina modificaron la dinámica macroeconómica y las políticas de empleo de las empresas, incidiendo en el desempeño del mercado de trabajo. El comportamiento de varones y mujeres difirió respecto al indicador agregado como la tasa de participación.

Superada la crisis de finales de la década de los 90, las mujeres mantuvieron relativamente elevadas las tasas de participación, es decir llegaron al mercado de trabajo para quedarse. Hacia el año 2007 se observó un mercado de trabajo con elevada inequidad de género y el ingreso 
Figura 5 Evolución de la Brecha Salarial en Uruguay, Período 1990-2018.

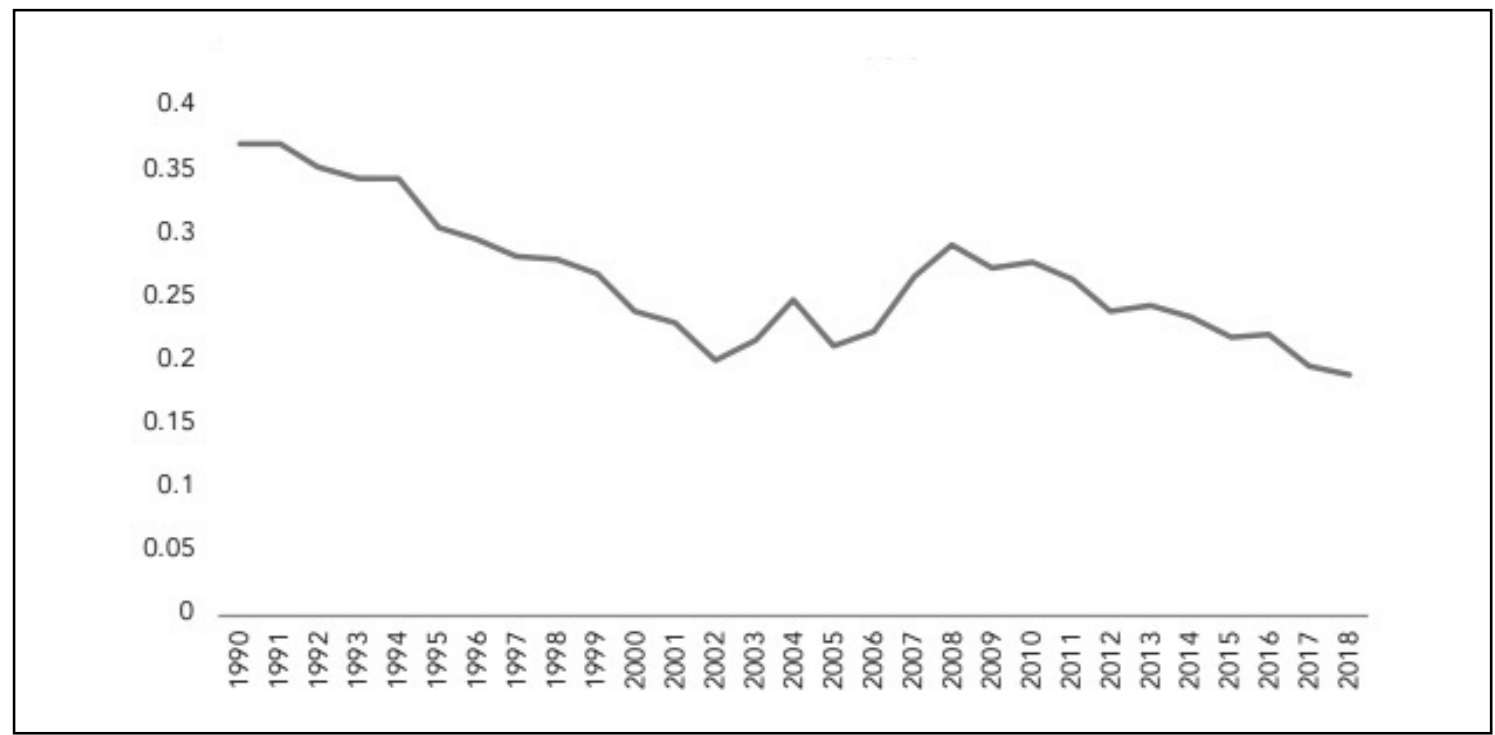

Fuente. Elaboración de la CEPAL hecha a partir de los datos obtenidos de la Encuesta Continua de Hogares $(\mathrm{ECH})$.

laboral de las mujeres es menor que el de los varones, producto de una inserción laboral más precaria: menor participación en el mercado de trabajo (Figura 4 ).

\section{Uruguay}

Según La Entidad de la ONU para la Igualdad de Género y el Empoderamiento de la Mujer (ONU Mujeres) las mujeres han incrementado su participación en la fuerza laboral en las últimas décadas. Sin embargo, las tasas de participación femenina se mantienen por debajo de las de los hombres y esto se mantiene para todas las edades y niveles educativos. Si bien los niveles de desempleo del país han bajado a niveles récord, los jóvenes, y especialmente las mujeres jóvenes, no tienen acceso a buenos puestos de trabajo. Mientras que el empleo informal ha disminuido en general, se ha mantenido ligeramente mayor entre las mujeres, especialmente en el interior del país.

La tasa de empleo más baja la presentan las mujeres que viven en hogares pobres $(38,6 \%)$, mientras que para varones que viven en hogares pobres se sitúa en
$64,2 \%$, con lo cual se genera una brecha de veinticinco puntos porcentuales. Finalmente, persiste la brecha salarial entre hombres y mujeres: el salario medio de las mujeres por hora trabajada fue de 11,1 puntos porcentuales inferior al de los hombres; paradójicamente, esta diferencia alcanzó su mayor magnitud entre las personas con los niveles educativos más altos: $27 \%$ (Colacce, s. f) (Figura 5).

\section{Conclusiones}

Es un hecho económico-social que la desigualdad de género afecta a todas las naciones y los Estados miembros del MERCOSUR no son excepciones, aunque puede afirmarse que las regulaciones formales de los mencionados Estados han avanzado para asegurar condiciones efectivas y favorables a fin de disminuir las brechas salariales entre hombres y mujeres, a pesar de que existen diversas convenciones de las Naciones Unidas como por ejemplo la Convención Nacional de Todas las Formas de Discriminación contra la Mujer, los Estados no necesariamente ajustan sus ordenamientos jurídicos $y$ 
legislaciones nacionales basándose en dichos instrumentos para mitigar la brecha existente e incluso aun existiendo una normativa nacional que impulse la igualdad de género no se desarrollan las medidas y estrategias necesarias para que las mencionadas normativas se cumplan en su totalidad.

Por un lado, podemos mencionar a Paraguay y a Brasil como los Estados con mayores brechas salariales entre los países estudiados ya que las políticas empleadas no se ajustan a la realidad de dichos países. Por otro lado, Argentina es el país con el menor Îndice de brecha salarial de la región puesto que las legislaciones implementadas favorecen la igualdad de oportunidades; siguiendo el ejemplo de Argentina, Uruguay ha reducido la brecha salarial en la última década debido a las estrategias legales que ha impulsado el Estado.

Por lo tanto, se concluye que es bastante importante y necesario luchar contra esta brecha fomentando el equilibrio de género en el mercado laboral cuya demanda cada vez va en aumento dentro de la sociedad redireccionando los roles de los hombres y las mujeres en dimensiones públicas como también privadas, haciendo énfasis en la igualdad de capacidades, siendo así el deber de la sociedad demostrar el valor de la igualdad a través de un trato justo y equivalente en la retribución del trabajo remunerado de la mujer.

\section{Referencia}

Altonji, J. G., \& Blank, R. M. (1999). Race and gender in the labor market. En Handbook of Labor Economics (Vols. 3, Part C, pp. 3143-3259). Elsevier. https://ideas.repec. org/h/eee/labchp/3-48.html

Araújo Freitas, A. (2015). La desigualdad salarial de género medida por regresión cuantílica: el impacto del capital humano, cultural y social. Revista mexicana de ciencias políticas y sociales, $60(223), 287-315$ http://www.scielo.org.mx/scielo. php?script $=$ sci_arttext $\&$ pid $=$ So18519182015000100o11\&lng=es\&tlng=es
Artza, L. L., Groh, M. J. C., Cabañas, A. C., \& Sánchez, A. (s. f.). La brecha salarial entre hombres y mujeres en América Latina. 110.

Brizuela, S., \& Tumini, L. (2008). Inequidades de género en el mercado de trabajo de la Argentina: Las brechas salariales. Revista de trabajo, 4(6).

CEPAL. (2017). 40 años de Agenda Regional de Género. CEPAL. https://www.cepal. org/es/publicaciones/40333-40-anosagenda-regional-genero

Colacce, M. (s. f.). Brechas de género en los ingresos laborales en el Uruguay. 59.

González, M., Halperin Weisburd, L., Horen, B., Labiaguerre, J. A., Müller, G., Sena, A. de, \& Villadeamigo, J. (2009). Cuestiones de género, mercado laboral y políticas sociales en América Latina: El caso argentino. CEPED. http:// www.econ.uba.ar/www/institutos/ economia/Ceped/publicaciones/dts/ DT\%2013\%20-\%2oHalperin\%2oet\%2O al.pdf

International Labour Office, \& Bureau for Gender Equality. (2013). Resumen ejecutivo: Trabajo decente e igualdad de género: políticas para mejorar el acceso y la calidad del empleo de las mujeres en América Latina y el Caribe. http://books.google.com/ books?id=J6jdjoCYCFoC

International Labour Office. (2010). Promoção da igualdade de oportunidades de gênero e raça no mundo do trabalho: Avanços e desafios. OIT.

Jabbaz, M., Gras, M., \& Díaz, C. (2019). La brecha salarial de género en las instituciones científicas. Estudio de caso. Convergencia Revista de Ciencias Sociales, 26, 1. https://doi.org/10.29101/ crcs.v26i8o.11248

Marchionni, M., Gasparini, L., \& Edo, M. (s. f.). Brechas de género en América Latina. Un estado de situación. https:// ideas.repec.org/b/dbl/dblbks/14o1. html

OIT, CEPAL, FAO, PNUD, \& ONUMUJERES. (2013). Trabajo decente e igualdad 
de género. Políticas para mejorar el acceso y la calidad del empleo en América Latina y el Caribe. http:// www.ilo.org/santiago/publicaciones/ WCMS_233161/lang--es/index.htm

OIT. (2005). Questionando um mito: Custos do trabalho de homens $e$ mulheres - International Labour Organization. https://labordoc. ilo.org/discovery/fulldisplay/ alma $993775443402676 / 41 \mathrm{ILO}$ INST:41ILO_V2

OIT. (2018). Avances y retos en reducir la brecha salarial y otras brechas de género en el marco de la Agenda 2030 para el Desarrollo Sostenible. https:// labordoc.ilo.org/discovery/fulldisplay/ alma $995074093302676 / 41$ ILO_ INST:41ILO_V2
Serafini, V., Egas, M. I. (2018). Empleo femenino en Paraguay: Tendencias y políticas públicas. https://mega.nz/\#! ZCxl2STZ!imV8g56SJOyjIhgHjDRvEJk NybMKGQZ-I9mmfFbezUo

Valenzuela, M. E., Reinecke, G. (200o). Más y mejores empleos para las mujeres? La experiencia de los países del Mercosur y Chile. Oficina Internacional del Trabajo.

Valverdi, N. M. O. (2017). Discriminación salarial: Brecha salarial entre hombres y mujeres del mercado laboral paraguayo. Población y Desarrollo, 44, 2-15.

Wereszczuk, M. G., \& Moreno, C. (s. f.). Participación de las mujeres y el techo de cristal en el sector financiero Argentino. 114.

\section{Sobre los Autores}

Ruth Romina Arleth Romero Vian

Estudiante de quinto año de la carrera de Economía por la Facultad de Ciencias Económicas de la Universidad Nacional de Asunción. Actividades extracurriculares: voluntaria en investigaciones de varias organizaciones no gubernamentales y Activista en Amnistía Internacional Paraguay.

\section{Marcelo Mathías Sánchez Zárate}

Estudiante de quinto año de la carrera de Economía por la Facultad de Ciencias Económicas de la Universidad Nacional de Asunción. Adjudicado con la Beca Itaipú a través del programa "Becas Itaipú", correspondiente a la promoción 2018.

\section{Mathías Federico González Acosta}

Estudiante de quinto año de la carrera de Economía por la Facultad de Ciencias Económicas de la Universidad Nacional de Asunción.

Actualmente trabajando como Técnico Catastral en el Servicio Nacional de Catastro como así también voluntario de varias organizaciones.

Antonella Cabral

Docente en la Universidad Nacional de Asunción, Facultad de Ciencias Económicas. 